\title{
Compassion fatigue among nurses working with older adults
}

\author{
Kay L.Kolthoff, Susan E.Hickman
}

\begin{abstract}
Nursing who care for older patients are exposed to significant suffering and loss that can lead to the development of compassion fatigue and burnout. An exploratory descriptive study was conducted to assess compassion fatigue, burnout, and compassion satisfaction in a group of 42 nurses who worked on a geriatric medicine unit using the Professional Quality of Life (ProQOL) Compassion Satisfaction and Compassion Fatigue 5 Scale. Nurses reported average levels of compassion fatigue, burnout, and compassion satisfaction. However, the compassion fatigue scores for newer nurses were higher $(\mathrm{x}=$ mean score $=28.7, \mathrm{std}=9.75)$ than the scores of more experienced nurses $(x=21.9, \mathrm{std}=4.66)(p<.01)$. Findings suggest the need to purposely build a supportive environment that focuses on new nurses to reduce compassion fatigue and burnout while enhancing compassion satisfaction.
\end{abstract}

Nurses who care for older adults are routinely exposed to suffering and loss in the course of caring for high needs patients. These experiences can result in the development of compassion fatigue that can make continuing to care for patients challenging. ${ }^{1}$ Compassion fatigue may occur in situations when the patient cannot be rescued or saved from harm, such as geriatric adults who experiences a loss of function, independence or death. This may result in the nurse feeling guilt or distress. ${ }^{2}$ Compassion fatigue, also known as secondary traumatic stress, is an emotional, physical and mental exhaustion. A person experiencing compassion fatigue feels depleted, chronically tired, helpless, hopeless and sad, even cynical about work, life, the state of the world and about oneself. ${ }^{3,4}$ Empathy and emotional energy are underlying drivers in the development of compassion fatigue. ${ }^{3}$ Individuals who suffer from compassion fatigue love what they do, but have difficulty maintaining emotional engagement with their patients.

The term compassion fatigue was first introduced in the early 1990s to describe situations where nurses had either turned off their own feelings or experienced helplessness and anger in response to the stress they feel watching patients go through devastating illnesses or trauma. ${ }^{5}$

This is the author's manuscript of the article published in final edited form as: Kolthoff, K. L., \& Hickman, S. E. (2017). Compassion fatigue among nurses working with older adults. Geriatric Nursing, 38(2), 106-109. https://doi.org/10.1016/j.gerinurse.2016.08.003 
The concept of compassion fatigue initially emerged from the field of nursing, though it can be experienced by other professions such as police officers, firefighters, and psychologists. ${ }^{4}$ According Potter, Deshields, and Rodriquez (2013), compassion fatigue is a prevalent condition. Recent research as shown that compassion fatigue is common among forensic nurses, psychologists, licensed social workers, and nurses who work in emergency departments, oncology, pediatrics and hospice. ${ }^{6}$ Other studies have demonstrated that psychiatrists, in particular have high rates of suicide, severe depression, and general compassion fatigue. This occupational hazard can have a profound effect on well-being. It may cause stress-related symptoms ${ }^{7}$ and is associated with fear, sleep difficulties, and avoidance. ${ }^{4}$ Accumulating research in nursing indicates that poor professional quality of life, which includes manifestations of both compassion fatigue and burnout, negatively impacts quality of care. ${ }^{8}$ Professional burnout is a related construct that results from a work environment that makes it difficult to achieve work goals and do ones job effectively. ${ }^{3,4}$ In contrast, compassion satisfaction is about out the enjoyment a nurse derives from being able to do his/her work well. ${ }^{4}$

This exploratory descriptive study of nurse caring for geriatric patients was designed to achieve the following goals: 1) determine the prevalence of compassion fatigue, burnout, and compassion satisfaction; 2) assess the association between compassion fatigue, burnout, and compassion satisfaction and years of professional experience. It is hypothesized that nurses with fewer years of experience who care for geriatric patients experiencing trauma, pain and suffering, nurses will have a higher levels of compassion fatigue and burnout in comparison to nurses who have worked with geriatric patients greater than one year. This study will fill a gap in literature by providing information factors about factors influencing compassion fatigues in nurses caring for geriatric patients. 


\section{METHODS}

\section{Setting}

This study was reviewed and approved by the Indiana University Institutional Review Board (IRBs) and the study sponsor IRB. The research was conducted in a Midwest non-for -profit faith-based 470 -bed acute care hospital, in a 35- private bed inpatient geriatric medicine unit.

\section{Sample}

The sample consisted of registered nurses (RNs). In order to be eligible for participation, nurses needed to meet the following inclusion criteria: employed full time or part time on the geriatric medicine unit; work at the bedside delivering direct patient care; and identify the unit as their primary unit. Middle management nurses, for example, charge nurses and patient care coordinators, who also deliver direct patient care, were also invited to participate in the study. Senior management was excluded.

\section{Procedures}

Data were collected between April 2014 and May 2014. Surveys and demographic questionnaires were placed in the mailboxes of all eligible participants. Each packet had preassigned study identification number. The master roster was kept in a locked file cabinet in a locked office, accessible only to the PI.

After completion of the survey and demographic questionnaire, participants were asked to place both items in a sealed envelope and drop it in a locked box located in the staff breakroom. Data were entered into SPSS and stored in a password protected file. The raw data was shared only with members of the research team.

\section{Data Collection Tools}


Participant Characteristics. General sociodemographics were collected from each nurse participant including gender, ethnicity, race, age, highest degree earned. Participants were also asked to provide information about their work responsibilities and years of experience. Nurses who have worked on the geriatric medicine unit for less than one year were identified as inexperienced nurses. Nurses who have worked on the geriatric medicine unit for greater than one year were identified as experienced nurses.

Professional Quality of Life 5 Scale. This 30 -item tool is used to measure compassion fatigue, professional burnout, and compassion satisfaction. ${ }^{3,4}$ The Professional Quality of Life (ProQOL) Scale ${ }^{4}$ evolved from Compassion Fatigue Self-Test. ${ }^{3}$ Participants were asked to respond to statements indicating how often they have experienced each situations described in the last 30 days using a Likert scale that ranges from 1 (never) to 5 (very often). Subscale scores are calculated by adding together individual item responses within each subscale (compassion fatigue, professional burnout, and compassion satisfaction). A subscale total score of $<22=10 w$; 23 to 41 = average; and $\geq 42=$ high. There is good construct validity with over 200 published papers. There is also more than 100,000 articles on the internet. Of the 100 published research papers on compassion fatigue, secondary traumatic stress and vicarious traumatization, nearly half have utilized the ProQOL or one of its earlier versions. The alpha reliability for each subscale is as follows: compassion fatigue $\alpha=.81$, burnout $\alpha=.65$, and compassion satisfaction $\alpha=.88 .{ }^{4}$ The 3 subscales measure separate constructs and show good construct validity (see Appendix A).

\section{Data Analysis}


Data were analyzed using SPSS v21.0. Descriptive statistics were used to describe sociodemographic variables. Independent $t$-tests were performed to compare the subscale scores of experienced versus inexperienced nurses. The $\mathrm{p}$ - value was set at $\mathrm{p}<.05$.

\section{RESULTS}

Overall, 42/50 (84\%) of surveys were returned. A majority of nurses who responded $(37 / 42$ or $88 \%)$ had one or more years of experience; a minority $(5 / 42)$ had less than a year of experience. Participant demographics are presented in Table 1.

The data for all participants $(n=42)$ were aggregated to determine the overall subscale scores for compassion fatigue, burnout, and compassion satisfaction. Subscales scores fell within the average range on each subscale (see Table 2).

Using the independent $t$-test, there was a significant difference in compassion fatigue scores between nurses who have worked on the geriatric medicine unit for less than one year (mean score $=28.7, \operatorname{std}=9.75)$ versus those who have worked on this unit greater than one year (mean score $=21.9, \operatorname{std}=4.66)(t=2.88, p=.006)$. There was also a significant difference in burnout scores between nurses who have worked on the geriatric medicine unit for less than one year (mean score $=31.6, \mathrm{std}=7.64)$ versus those who have worked there greater than one year (mean score $=25.7, \operatorname{std}=5.38)(t=2.45, p=.019)$. The findings of this study suggest that nurses with fewer years of experience on the geriatric medicine unit have higher levels of compassion fatigue and burnout in comparison to nurses who have worked there greater than one year. However there was no significant difference in compassion satisfaction scores between nurses who have more experience $($ mean score $=37.6, \mathrm{std}=4.58)$ versus those with less experience $($ mean score $=37.3$, $\operatorname{std}=5.45)(t=.119, p=.906)$.

\section{DISCUSSION}


The findings of this study suggest that nurses with fewer years of experience on the geriatric medicine unit have higher levels of compassion fatigue and burnout in comparison to nurses who have worked there greater than one year. It has been reported in the literature that the less experienced nurse who brings high expectations to a job has been found to be at risk for compassion fatigue. ${ }^{9}$ Less experienced nurses may be more vulnerable to compassion fatigue because they have not yet developed a peer support group or because they may fail to incorporate activities that renew or re-energize them to help better respond to the needs of others. These nurses may also be more prone to work extra shifts and fail to set realistic boundaries and timelines to complete necessary tasks, increasing their risk of burnout. ${ }^{9}$ More experienced nurses have had time to develop professional resiliency skills to be maximally functional under the emotional demands of their work environment. ${ }^{3}$

Despite the significant differences in compassion fatigue and burnout between newer versus more experienced nurses, the overall subscale scores of compassion fatigue and burnout were in the average range. There were also no differences in compassion satisfaction for less versus more experienced nurses. These results indicate these nurses experience a sense of satisfaction and achievement in their ability to empathize and to relieve suffering without getting emotionally overwhelmed. ${ }^{3}$ These findings also suggest that the geriatric medicine nurses are able to connect with patients they care for regardless of the outlook and may lead to purposeful and meaningful interactions between the nurse and their patients. ${ }^{10}$

Findings suggest the need to purposely build a supportive environment that prevents professional burnout and compassion fatigue and sustain compassion satisfaction. Department values as expressed through policies and procedures should support staff in balancing work and personal needs, as well as provide for adequate time off, assignment rotations, flexible 
scheduling, education benefits, and other self- care and development activities. ${ }^{9}$ Many compassion fatigue interventions are free, such as management actively listening to their staff. Self-care is a priority in trauma informed initiatives because the effects of working with the geriatric population can take its toll.

Optimally, organizations should provide preventative and proactive support services instead of in response to a difficult case or crisis. For example, Barnes-Jewish Hospital has developed a systemic program for compassion fatigue resiliency. The implementation of a hospital wide resiliency program is designed to help professional caregivers understand compassion fatigue, recognize the physical, mental and emotional effects of stress, and adopt resiliency strategies. The compassion fatigue resiliency program is based on Dr. J. Eric Gentry Accelerated Recovery Program which is a treatment protocol for professionals who experience the effects of compassion fatigue. This voluntary program has been well received by participants and post evaluation surveys show promising results with a decline in compassion fatigue and burnout among participants. ${ }^{6}$

Compassion fatigue prevention and screening programs should ideally be developed for nurses and other frontline employees. ${ }^{2}$ If nurses are provided with the screening scale for early identification and recognition of compassion fatigue, it is possible it will enhance long term retention, increase nursing engagement and promote professional development. ${ }^{9}$ Periodic rescreening is important to monitor progress over time. ${ }^{9}$

Geriatric nurses and nurse executives should be mindful that burnout and compassion fatigue affects not only individual performance, but also that of the work group. ${ }^{8}$ One potentially important use of the information is to reevaluate and adjust one's self care plan or even a groups' or organization's plan. According to the American Nurses Association Code of Ethics Provision 
5 , nurses have a responsibility to try to reduce the effects of compassion fatigue through a healthy lifestyle that supports their own well-being. ${ }^{11}$ Similarly, nurses have the responsibility to intervene, support, and help each other in recognizing and addressing the signs and symptoms of burnout and compassion fatigue. Nurse executives should focus on strategies that emphasize relationship-centered values and promote a culture of caring that recognizes the extraordinary acts of staff with patient on a day- to- day basis.

\section{Limitations}

There are several limitations to this study. First, the findings of this study reflect the scores of convenience sample of nurses and overall number of inexperienced nurses participating was low $(n=5)$. Second, this study was conducted on one unit at one suburban non-for -profit faith-based health care system that is pursuing Magnet designation, so the results may not be generalizable. Third, the population lacked diversity so the results may not be generalizable to more diverse settings. Finally, the study measured compassion fatigue, compassion satisfaction, and burnout at a single point in time with no long- term follow-up. It is likely that perceptions change over time due to changes in individual and workplace circumstances. ${ }^{8}$

\section{Conclusion}

Study findings indicate that this group of geriatric nurses experience average levels of compassion fatigue, burnout, and compassion satisfaction. However, there were significant differences in the levels of compassion fatigue and burnout for nurses with less than one year of experience in comparison to nurse with more than one year of experience. Data was disseminated to the nursing shared governance unit based staff council to help develop and promote creative unit based compassion fatigue interventions to address these burgeoning threats to job satisfaction. Sustained compassion satisfaction in the new and experienced geriatric 
medicine nurses will possibly help with nurse retention. The personal and organizational benefits of compassion fatigue interventions will occur over an extended time, on more than one occasion, with results that may not appear immediately and may be incremental and cumulative rather than sudden and dramatic. ${ }^{9}$ These findings of this study underscore the importance of adopting a geriatric medicine compassion fatigue resiliency program.” Future research should explore the effect of regularly screening nurses and its effect on long-term retention, enhanced nursing engagement and professional development will be the outcomes. These interventions may be particularly beneficial for new nurses.

\section{FUNDING}

This study was conducted as part of the requirements for the Doctorate of Nursing Practice. The principal investigator and co-investigator had full access to the data and was responsible for the study protocol, statistical analysis plan, study progress, analysis, study reporting. 


\section{REFERENCES}

1. Figley CR. Treating compassion fatigue. Hove, Great Britain: Brunner-Routledge; 2002.

2. Yoder EA. Compassion fatigue in nurses. Applied Nursing Research 2010; 23: 191-197.

3. Figley CR. Compassion fatigue: Coping with secondary traumatic stress disorder in those who treat the traumatized. New York, NY: Brunner/ Mazel; 1995.

4. Stamm BH. The ProQOL concise Manual (2nd ed.). http://www.ProQOL.org; 2010 Accessed 12.05.16.

5. Joinson C. Coping with compassion fatigue. Nursing, 2010;22(1):116-121.

6. Potter P, Deshields T, Rodriquez S developing a systemic program for compassion fatigue. Nursing Administration 201337(4):326-332.

7. Landro L. When nurses catch compassion fatigue, patients suffer. The Wall Street Journal. http://online.wsj.com; 3.1.12 Accessed 12.05.16.

8. Todaro-Franceschi V. Critical care nurses' perceptions of preparedness and ability to care for the dying and their professional quality of life. Dimensions in Critical Care 2013;32(4):184-190.

9. Aycock N, Boyle D. Interventions to manage compassion fatigue in oncology nurses. Clinical Journal of Oncology Nursing 2009;13(2):183-191.

10. Young JL, Derr DM, Cicchillo VJ, Bressler S. Compassion satisfaction, burnout, and secondary traumatic stress in heart and vascular nurses. Critical Care Nursing 2011; 34(3):227-234. 
11. American Nurses Association. Code of Ethics for Nurses with interpretative statements. http://www.nursingworld.org/DocumentVault/Ethics 1/Code-of-Ethicsfor-Nurses.html 2015 Accessed 12.05.16. 
Table 1. Demographic variables for Geriatric Nurse Participants.

\begin{tabular}{lc} 
Variable & $\mathrm{n}=42$ \\
\hline Age (years) & $\mathrm{n}(\%)$ \\
$20-29$ & $8(19 \%)$ \\
$30-39$ & $10(24 \%)$ \\
40 or older & $24(57 \%)$ \\
& \\
Highest level of nursing education & $19(45 \%)$ \\
Associate & $3(8 \%)$ \\
Diploma & $19(45 \%)$ \\
Bachelors & $1(2 \%)$ \\
Masters &
\end{tabular}

Years of experience as RN on the Center for Geriatric Medicine

$<$ Than 1 year

$5(12 \%)$

$>$ Than 1 year

$37(88 \%)$

Gender

Male

$5(12 \%)$

Female

$37(88 \%)$

Hours per week worked on the Center for Geriatric Medicine 
Hours per week worked outside of the Center for Geriatric Medicine

None

$<20$

$>20$

Primary position

Staff nurse

Management

Preceptor

Yes

No

Ethnicity

Hispanic

Non-Hispanic

Race

Black/African American

White/Caucasian
$37(88 \%)$

$2(5 \%)$

$3(7 \%)$

$40(95 \%)$

$2(5 \%)$

$15(36 \%)$

$27(64 \%)$

$0(0 \%)$

$42(100 \%)$

$7(17 \%)$

$32(76 \%)$ 
Professional Quality of Life

Asian

Other
$2(5 \%)$

$1(2 \%)$ 
Table 2. The Professional Quality of Life compassion fatigue, professional burnout, and compassion satisfaction scores for new and experienced nurses.

\begin{tabular}{|c|c|c|c|}
\hline ITEM & $\begin{array}{l}\text { New Nurses } \\
(n=5)\end{array}$ & $\begin{array}{l}\text { Experienced } \\
\text { Nurses } \\
(n=37)\end{array}$ & $\begin{array}{l}\text { Total } \\
(\mathrm{n}=42)\end{array}$ \\
\hline \multicolumn{4}{|c|}{ COMPASSION FATIGUE SUBSCALE } \\
\hline $\begin{array}{l}\text { I am preoccupied with more than one } \\
\text { person I [help]. }\end{array}$ & 3.8 & 3.5 & 3.7 \\
\hline $\begin{array}{l}\text { I jump or am startled by unexpected } \\
\text { sounds. }\end{array}$ & 3.7 & 2.6 & 3.2 \\
\hline $\begin{array}{l}\text { I find it difficult to separate my personal life } \\
\text { from my life as a [helper]. }\end{array}$ & 2.5 & 2.3 & 3.2 \\
\hline $\begin{array}{l}\text { I think that I might have been affected by } \\
\text { the traumatic stress of those I [help]. }\end{array}$ & 2.7 & 1.2 & 2.4 \\
\hline $\begin{array}{l}\text { Because of my [helping], I have felt "on } \\
\text { edge" about various things. }\end{array}$ & 3.0 & 2.5 & 2.0 \\
\hline $\begin{array}{l}\text { I feel depressed because of the traumatic } \\
\text { experiences of the people I [help]. }\end{array}$ & 2.5 & 2.0 & 2.3 \\
\hline
\end{tabular}




\begin{tabular}{|c|c|c|c|}
\hline $\begin{array}{l}\text { I feel as though I am experiencing the } \\
\text { trauma of someone I have [helped]. }\end{array}$ & 2.7 & 1.8 & 2.3 \\
\hline $\begin{array}{l}\text { I avoid certain activities or situations } \\
\text { because they remind me of frightening } \\
\text { experiences of the people I [help]. }\end{array}$ & 2.3 & 1.6 & 2 \\
\hline $\begin{array}{l}\text { As a result of my [helping], I have } \\
\text { intrusive, frightening thoughts. }\end{array}$ & 1.5 & 1.6 & 2 \\
\hline $\begin{array}{l}\text { I can't recall important parts of my work } \\
\text { with trauma victims }\end{array}$ & 3.7 & 2 & 2.9 \\
\hline $\begin{array}{l}\text { AVERAGE COMPASSION } \\
\text { FATIGUE SCORE (STD) }\end{array}$ & $\begin{array}{l}28.7 \\
(9.8) * *\end{array}$ & $21.9(4.8)^{* *}$ & $23.02(6.2)$ \\
\hline \multicolumn{4}{|c|}{ BURNOUT SUBSCALE } \\
\hline I am happy. Item is reversed scored. & 2.5 & 2.1 & 2.3 \\
\hline $\begin{array}{l}\text { I feel connected to others. Item is reversed } \\
\text { scored. }\end{array}$ & 2.3 & 2 & 2.2 \\
\hline $\begin{array}{l}\text { I am not as productive at work because I am } \\
\text { losing sleep over traumatic experiences of a } \\
\text { person I [help]. }\end{array}$ & 1.8 & 1.75 & 1.8 \\
\hline I feel trapped by my job as a [helper]. & 2.8 & 2.3 & 2.6 \\
\hline
\end{tabular}




\begin{tabular}{|c|c|c|c|}
\hline $\begin{array}{l}\text { I have beliefs that sustain me. Item is } \\
\text { reversed scored. }\end{array}$ & 2.2 & 2.8 & 2.5 \\
\hline $\begin{array}{l}\text { I am the person I always wanted to be. } \\
\text { Item is reversed scored. }\end{array}$ & 2.3 & 3.2 & 2.8 \\
\hline $\begin{array}{l}\text { I feel worn out because of my work as a } \\
\text { [helper]. }\end{array}$ & 4.2 & 3.8 & 4 \\
\hline $\begin{array}{l}\text { I feel overwhelmed because my case [work] } \\
\text { load seems endless. }\end{array}$ & 4 & 3.7 & 3.9 \\
\hline I feel "bogged down" by the system. & 4 & 3.2 & 3.6 \\
\hline $\begin{array}{l}\text { I am a very caring person. Item is reversed } \\
\text { scored. }\end{array}$ & 1.3 & 1.6 & 1.5 \\
\hline $\begin{array}{l}\text { AVERAGE BURNOUT } \\
\text { SCORE (STD) }\end{array}$ & $31.6(7.6)^{*}$ & $25.7(5.4)^{*}$ & $26.69(6.1)$ \\
\hline \multicolumn{4}{|c|}{ COMPASSION SATISFACTION SUBSCALE } \\
\hline $\begin{array}{l}\text { I get satisfaction from being able to [help] } \\
\text { people }\end{array}$ & 4.5 & 4.4 & 4.5 \\
\hline $\begin{array}{l}\text { I feel invigorated after working with those I } \\
\text { [help]. }\end{array}$ & 3.7 & 3.1 & 3.4 \\
\hline
\end{tabular}




\begin{tabular}{|c|c|c|c|}
\hline I like my work as a [helper]. & 3.8 & 3.9 & 3.9 \\
\hline $\begin{array}{l}\text { I am pleased with how I am able to keep up } \\
\text { with [helping] techniques and protocols. }\end{array}$ & 3 & 3.4 & 3.2 \\
\hline My work makes me feel satisfied. & 3.2 & 3.4 & 3.3 \\
\hline $\begin{array}{l}\text { I have happy thoughts and feelings about } \\
\text { those I [help] and how I could help them. }\end{array}$ & 4.0 & 3.5 & 3.8 \\
\hline $\begin{array}{l}\text { I believe I can make a difference through } \\
\text { my work. }\end{array}$ & 4.3 & 3.8 & 4.1 \\
\hline I am proud of what I can do to [help]. & 4.2 & 3.1 & 3.7 \\
\hline $\begin{array}{l}\text { I have thoughts that I am a "success" as a } \\
\text { [helper] }\end{array}$ & 3.7 & 3.4 & 3.6 \\
\hline I am happy that I chose to do this work & 3.9 & 4 & 4 \\
\hline $\begin{array}{r}\text { AVERAGE COMPASSION } \\
\text { SATISFACTION SCORE (STD) }\end{array}$ & $37.6(4.6)$ & $37.3(5.5)$ & $37.4(5.2)$ \\
\hline
\end{tabular}

Note: Participants were asked to rate each item using the following scale: $1=$ Never 2=Rarely $3=$ Sometimes $4=$ Often $5=$ Very Often.

* Indicates significant differences $(\mathrm{p}<.05)$ between new and experienced nurses.

** Indicates significant differences $(\mathrm{p}<.01)$ between new and experienced nurses. 
Reference:

Stamm, B.H.(2010). The ProQOL concise Manual (2nd ed.). Retrieved from http://www.ProQOL.org 